\title{
Calibrating a solar x-ray telescope using the Crab Nebula
}

\section{Karl Klett Jr. and Steven Hill}

Summed observations of a well-studied astronomical $x$-ray source can provide an inexpensive alternative to data collected with soundingrocket underflights for calibrating space-based solar telescopes.

The Crab Nebula (see Figure 1) has been used to calibrate $x$ ray detectors on many satellite-, rocket-, and balloon-borne missions. It provides a bright and stable broadband source with a well-characterized spectrum. For space-based telescopes, in particular, this allows adjusting to changes over time in filters, electronics, optics, and other sensors. ${ }^{1}$ We demonstrate here the feasibility of extending this calibration practice to the Solar XRay Imager (SXI-N) telescope aboard a Geostationary Operational Environmental Satellites (GOES) mission launched in May $2006 .^{2}$

The primary mission of the GOES series, which is operated by the National Oceanic and Atmospheric Administration (NOAA), is to monitor weather on Earth. SXI's purpose is to provide real-time images of the sun and solar corona between 6 and $60 \AA$, which helps space weather forecasters by providing longterm solar climatological observations.

Previous SXI instruments were not calibrated. They suffer from ongoing filter and electronic unit degradation, and a consequent degree of measurement uncertainty. Onboard x-ray calibration of satellite instruments is not generally practical because the required sources would be too large. NOAA's current plan for calibrating SXI-N would use expensive sounding-rocket underflights to make solar observations simultaneously with the GOES. ${ }^{3}$

In-flight x-ray calibrations using the Crab Nebula may not have been considered in these cases because of the nebula's low flux compared with that of the solar phenomena being measured. To evaluate the feasibility of this approach, we used existing Crab Nebula measurements from the Einstein Observatory, shown in Figure $2{ }^{4}$ to see whether SXI-N observations could achieve a signal-to-noise ratio (SNR) of at least 5, which would meet the instrument's 20\% photometric requirement. We also modeled likely noise sources that would enter into our signalto-noise calculations.

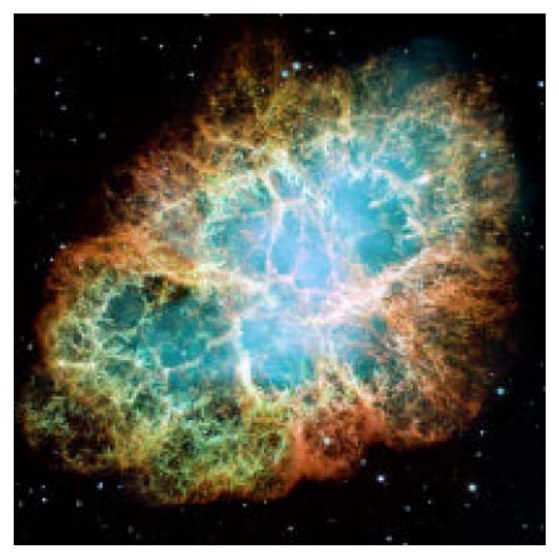

Figure 1. The Crab Nebula, shown at visible wavelengths. Courtesy of NASA and ESA (Hubble WFPC2), and Allison Loll and Jeff Hester (Arizona State University).

The conversion of the nebula's photon spectrum $F_{x-\text { ray }}(\lambda)$ into signal electrons $S_{x-\text { ray }}$ generated by SXI-N's CCD was modeled as the following integral over wavelength $\lambda$,

$$
\begin{aligned}
S_{x-\text { ray }}=\int_{9 \AA}^{30 \AA} \quad & F_{x-r a y}(\lambda) \cdot b(\lambda) \cdot e f(\lambda) \cdot A_{\text {eff }}(\lambda) . \\
& F i l(\lambda) \cdot c(\lambda) \cdot Q E_{C C D}(\lambda) d \lambda
\end{aligned}
$$

where:

$S_{x-\text { ray }} \quad$ Signal electrons generated in the CCD by Crab

$F_{x-\text { ray }}(\lambda)$

Nebula x-rays.

photons $\mathrm{s}^{-1} \mathrm{~cm}^{-2} \mathrm{keV}^{-1}$.

$b(\lambda) \quad$ Bandwidth conversion, from photons $/ \mathrm{keV}$ to photons/ $\mathrm{A}$.

ef $(\lambda) \quad$ Entrance filter transmission.

$A_{\text {eff }}(\lambda) \quad$ Grazing-incident mirror's effective area.

Fil $(\lambda) \quad$ Filter transmission.

$c(\lambda) \quad$ Electrons generated per $x$-ray photon at $\lambda$.

$Q E_{C C D}(\lambda) \quad C C D$ quantum efficiency. 


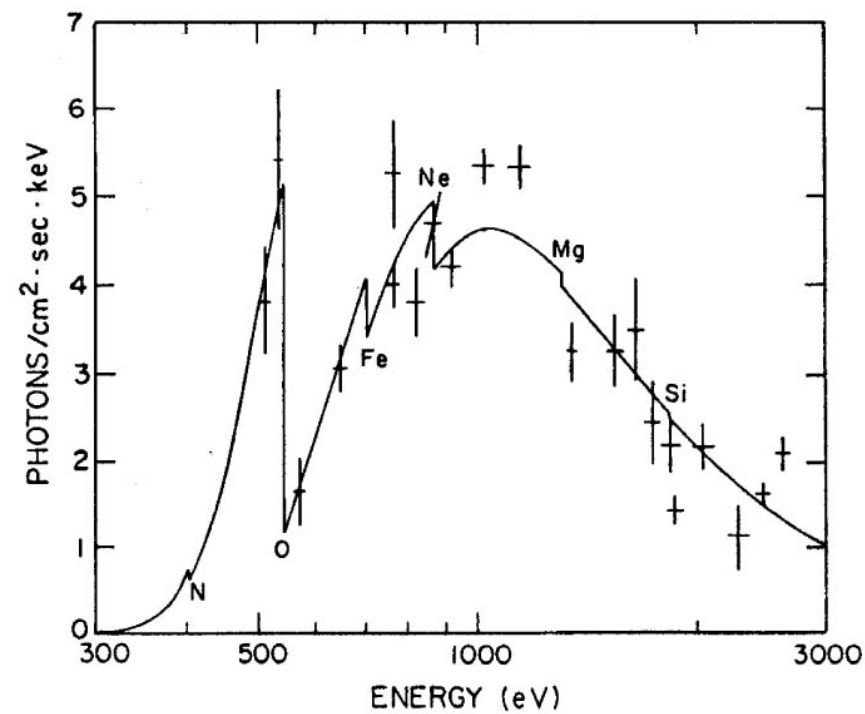

Figure 2. Measurements of the Crab Nebula $x$-ray flux spectrum (crosshairs) are shown, along with a model that incorporates interstellar medium absorption by several elements. ${ }^{4}$ Crosshairs give the bandwidth (horizontal bar) and error (vertical bar) of each measurement.

\begin{tabular}{|c|c|c|}
\hline & Thin Polymide & Be 12 \\
\hline SNR & 3.15 & 2.22 \\
\hline $\begin{array}{c}\text { X-Ray Photons } \\
\text { Detected }\end{array}$ & 76 & 11 \\
\hline $\begin{array}{c}\text { Images Required } \\
\text { for SNR=10 }\end{array}$ & 10 & 21 \\
\hline
\end{tabular}

Table 1. Shown are the estimated SNRs for SXI-N based on $65 \mathrm{~s}$ Crab Nebula calibration images.

The integration was performed numerically using a Microsoft Excel spreadsheet.

In computing the SNR, the total measurement uncertainty was assumed to be dominated by errors associated with photon counting (Poisson-distributed), CCD thermal noise, digitizer quantization error, and CCD readout noise.

An SNR of 5 'just' meets the photometric requirement, allowing for no performance degradation over time. An improved approach would be to sum several images, which should increase the ratio by the square root of the number of images summed. Using the SXI-N's longest exposure (65s) with the most ('Thin Polymide') and least ('Be 12') transmissive filters, a doubled SNR of 10 would require the sum of 10 and 21 images, respectively (see Table 1), which represent fairly modest operational demands.

This analysis of calibrating SXI-N using the Crab Nebula shows that effective calibration could be carried out with relatively small operational and analysis costs. Expensive calibration underflights, requiring sounding rockets, would not be needed. If successful, this calibration technique could be used by other space-based instruments that observe the sun at x-ray wavelengths.

\section{Author Information}

\section{Karl Klett Jr.}

Optics Group

Swales Aerospace

Beltsville, MD

Karl Klett Jr. attended his first SPIE meeting this past August, where he presented a paper that is the basis for this article.

\section{Steven Hill}

NOAA Space Environment Center

Boulder, $\mathrm{CO}$

Steven Hill is the SXI team lead at NOAA's Space Environment Center. He has published numerous papers in the field of space weather.

\section{References}

1. M. G. F. Kirsch et al., Crab: the standard $x$-ray candle with all (modern) $x$-ray satellites, Proc. SPIE 5898, pp. 22-43, 2005. doi:10.1117/12.616893

2. K. Klett and S. Hill, GOES N-P SXI in-flight calibration using the Crab Nebula, Proc. SPIE 6317, pp. 9-19, 2006. doi:10.1117/12/680800

3. S. Hill et al., The NOAA GOES $x$-ray calibration program, Proc. SPIE 5901, pp. 99112, 2005. doi:10.1117/12.619109

4. M. L. Schattenburg and C. R. Canizares, High-resolution $x$-ray spectroscopy of the Crab Nebula and the oxygen abundance of the interstellar medium, Astrophys. J. 301, pp. 759-771, 1986. doi:10.1086/163942 